\title{
Biosimilar candidate IBI305 plus paclitaxel/carboplatin for the treatment of non-squamous non-small cell lung cancer
}

\author{
Yunpeng Yang ${ }^{1}$, Bin $\mathrm{Wu}^{2}$, Linian Huang ${ }^{3}$, Meiqi Shi ${ }^{4}$, Yunpeng Liu ${ }^{5}$, Yanqiu Zhao ${ }^{6}$, Lijun $\mathrm{Wang}^{7}$, Shun $\mathrm{Lu}^{8}$, \\ Gongyan Chen ${ }^{9}$, Baolan $\mathrm{Li}^{10}$, Conghua Xie ${ }^{11}$, Jian Fang ${ }^{12}$, Nong Yang ${ }^{13}$, Yiping Zhang ${ }^{14}$, Jiuwei Cui ${ }^{15}$, \\ Yong Song ${ }^{16}$, Cuiying Zhang ${ }^{17}$, Xiaodong $\mathrm{Mei}^{18}{ }^{18}$, Bangwei Cao ${ }^{19}$, Lan Yang ${ }^{20}$, Ying Cheng ${ }^{21}$, Kejing Ying ${ }^{22}$, \\ Tao Sun ${ }^{23}$, Biyong Ren ${ }^{24}$, Qitao Yu ${ }^{25}$, Zijun Liao ${ }^{26}$, Zhidong Pei ${ }^{27}$, Mengzhao Wang ${ }^{28}$, Jianying Zhou ${ }^{29}$, \\ Shiying Yu ${ }^{30}$, Guosheng Feng ${ }^{31}$, Huiping Wan ${ }^{32}$, Huaqing Wang ${ }^{33}$, Shegan Gao ${ }^{34}$, Jinliang Wang ${ }^{35}$, \\ Guangyu $\mathrm{An}^{36}$, Yi Geng ${ }^{37}$, Yanxia Ji ${ }^{38}$, Ying Yuan ${ }^{39}$, Shenglin $\mathrm{Ma}^{40}$, Zhongyao Jia ${ }^{41}$, Mu Hu ${ }^{42}$, Hui Zhou ${ }^{43}$, \\ Jie Yu ${ }^{43}$, Xing Sun ${ }^{43}$, Li Zhang ${ }^{1}$
}

${ }^{1}$ Department of Medical Oncology, State Key Laboratory of Oncology in South China, Collaborative Innovation Center for Cancer Medicine, Sun Yat-sen University Cancer Center, Guangzhou 510000, China; ${ }^{2}$ Department of Respiratory Medicine, Affiliated Hospital of Guangdong Medical University, Zhanjiang 524002, China; ${ }^{3}$ Department of Respiratory Medicine, The First Affiliated Hospital of Bengbu Medical College, Bangbu 233000, China; ${ }^{4}$ Department of Oncology, Jiangsu Cancer Hospital, Nanjing 210009, China; ${ }^{5}$ Department of Oncology, The First Hospital of China Medical University, Shenyang 110001, China; ${ }^{6}$ Department of Respiratory Medicine, Henan Cancer Hospital, Zhengzhou 450003, China; ${ }^{7}$ Department of Tumor radiotherapy, The Second Affiliated Hospital of Xingtai Medical College, Xingtai 054000, China; ${ }^{8}$ Department of Oncology, Shanghai Chest Hospital, Shanghai 200030, China; ${ }^{9}$ Department of Oncology, Harbin Medical University Cancer Hospital, Harbin 150081 , China; ${ }^{10}$ General Department, Beijing Chest Hospital, Capital Medical University, Beijing 101149, China; ${ }^{11}$ Department of Oncology, Zhongnan Hospital of Wuhan University, Wuhan 430071, China; ${ }^{12}$ Department of Oncology, Peking University Cancer Hospital, Beijing 100142, China; ${ }^{13}$ Department of Pulmonary Gastroenterology, Hunan Cancer Hospital, Changsha 410006, China; ${ }^{14}$ Department of Oncology, Zhejiang Cancer Hospital, Hangzhou 310022, China; ${ }^{15}$ Department of Oncology, The First Hospital of Jilin University, Changchun 130000 , China; ${ }^{16}$ Department of Respiratory Medicine, General Hospital of Eastern Theater Command, Nanjing 210002, China; ${ }^{17}$ Department of Oncology, Inner Mongolia People's Hospital, Hohhot 010017, China; ${ }^{18}$ Department of Respiratory Medicine, An Hui Provincial Hospital, Hefei 230000, China; ${ }^{19}$ Department of Oncology, Peking Friendship Hospital, Capital Medical University, Beijing 100050, China; ${ }^{20}$ Department of Respiratory Medicine, The First Affiliated Hospital of Xi'an Jiaotong University, Xi'an 710061, China; ${ }^{21}$ Department of Oncology, Jilin Cancer Hospital, Changchun 130000, China; ${ }^{22}$ Department of Respiratory Medicine, Sir Run Shaw Hospital of Zhejiang University School of Medicine, Hangzhou 310016, China; ${ }^{23}$ Department of Internal Medicine, Liaoning Cancer Hospital \& Institute, Shenyang 110046, China; ${ }^{24}$ Department of Respiratory Medicine, Chongqing three Gorges Central Hospital, Chongqing 404000, China; ${ }^{25}$ Department of Oncology, The Cancer Hospital of Guangxi Zhuang Autonomous Region, Nanning 530000, China; ${ }^{26}$ Department of Internal Medicine, Shaanxi Provincial Tumor Hospital, Xi'an 710061, China; ${ }^{27}$ Department of Oncology, Luoyang Central Hospital Affiliated To Zhengzhou University, Luoyang 471009, China; ${ }^{28}$ Department of Respiratory Medicine, Peking Union Medical College Hospital, Beijing 100005, China; ${ }^{29}$ Department of Respiratory Medicine, The First Affiliated Hospital Zhejiang University, Hangzhou 310003, China; ${ }^{30}$ Department of Oncology, Tongji Hospital Tongji Medical College, Huazhong University of Science and Technology, Wuhan 430000, China; ${ }^{31}$ Department of Chemotherapy, The People's Hospital of Guangxi Zhuang Autonomous Region, Nanning 530000, China; ${ }^{32}$ Department of Oncology, Jiangxi People's Hospital, Nanchang 330000, China; ${ }^{33}$ Department of Oncology, Tianjin People's Hospital, Tianjin 300121, China; ${ }^{34}$ Department of Oncology, First Affiliated Hospital of Henan University of Science and Technology, Luoyang 471000, China; ${ }^{35}$ Department of Oncology, General Hospital of PLA, Beijing 100853, China; ${ }^{36}$ Department of Oncology, Beijing Chao-Yang Hospital, Beijing 100020, China; ${ }^{37}$ Department of Oncology, Baoji Central Hospital, Baoji 721008, China; ${ }^{38}$ Department of Oncology, Handan Central Hospital, Handan 056001, China; ${ }^{39}$ Department of Oncology, The Second Affiliated Hospital Zhejiang University, Hangzhou 310009, China; ${ }^{40}$ Department of Oncology, Hangzhou First People's Hospital, Hangzhou 310002, China; ${ }^{41}$ Department of Oncology, Linyi People's Hospital, Linyi 276003, China; ${ }^{42}$ Department of Chest Surgery, Xuanwu Hospital Capital Medical University, Beijing 100053, China; ${ }^{43}$ Innovent Biologics, Inc., Suzhou 215123 , China

Contributions: (I) Conception and design: L Zhang, H Zhou; (II) Administrative support: None; (III) Provision of study materials or patients: Y Yang, B Wu, L Huang, M Shi, Y Liu, Y Zhao, L Wang, S Lu, G Chen, B Li, C Xie, J Fang, N Yang, Y Zhang, J Cui, Y Song, C Zhang, X Mei, B Cao, L Yang, Y Cheng, K Ying, T Sun, B Ren, Q Yu, Z Liao, Z Pei, M Wang, J Zhou, S Yu, G Feng, H Wan, H Wang, S Gao, J Wang, G An, Y Geng, Y Ji, Y Yuan, S Ma, Z Jia, M Hu, L Zhang; (IV) Collection and assembly of data: Y Yang, B Wu, L Huang, M Shi, Y Liu, Y Zhao, L Wang, S Lu, 
G Chen, B Li, C Xie, J Fang, N Yang, Y Zhang, J Cui, Y Song, C Zhang, X Mei, B Cao, L Yang, Y Cheng, K Ying, T Sun, B Ren, Q Yu, Z Liao, Z Pei, M Wang, J Zhou, S Yu, G Feng, H Wan, H Wang, S Gao, J Wang, G An, Y Geng, Y Ji, Y Yuan, S Ma, Z Jia, M Hu, L Zhang; (V) Data analysis and interpretation: L Zhang, Y Yang, H Zhou, J Yu; (VI) Manuscript writing: All authors; (VII) Final approval of manuscript: All authors.

Correspondence to: Li Zhang. Department of Medical Oncology, State Key Laboratory of Oncology in South China, Collaborative Innovation Center for Cancer Medicine, Sun Yat-sen University Cancer Center, Guangzhou 510000, China. Email: zhangli@sysucc.org.cn.

Background: Bevacizumab is a monoclonal antibody $(\mathrm{mAb})$ against vascular endothelial growth factor (VEGF) and used for treatments of various cancers. Due to the high costs of bevacizumab treatments, a biosimilar provides an affordable alternative therapy for cancer patients.

Methods: In this randomized, double-blind, multicenter, phase 3 study, locally advanced, metastatic or recurrent non-squamous non-small cell lung cancer (NSCLC) patients with wild-type epidermal growth factor receptor were enrolled and randomized (1:1) into IBI305 or bevacizumab groups. Patients received 6 cycles of paclitaxel/carboplatin plus IBI305 or bevacizumab $15 \mathrm{mg} / \mathrm{kg}$ intravenously followed by IBI305 or bevacizumab $7.5 \mathrm{mg} / \mathrm{kg}$ maintenance until disease progression, unacceptable toxicity or death. The primary endpoint was confirmed objective response rate (ORR) by an independent radiological review committee (IRRC) and secondary endpoints included disease control rate (DCR), progression-free survival (PFS), duration of response (DOR), overall survival (OS) and safety.

Results: A total of 450 NSCLC patients were enrolled (224 in IBI305 group and 226 in bevacizumab group). ORRs were $44.3 \%$ for IBI305 and $46.4 \%$ for bevacizumab, and the ORR ratio was 0.95 (90\% CI: 0.803 to 1.135 ), within the predefined equivalence margin of 0.75 to 1.33 . No significant difference in PFS (7.64 vs. $7.77 \mathrm{~m}, \mathrm{P}=0.9987$ ) was observed between the 2 groups. Serious adverse events (AEs) occurred in $33.5 \%(75 / 224)$ of patients in the IBI305 group and 37.6\% (85/226) in the bevacizumab group. AEs $\geq$ grade 3 were similar in the IBI305 and bevacizumab groups [84.4\% (189/224) vs. 89.8\% (203/226), P=0.085].

Conclusions: IBI305 is similar to bevacizumab in terms of efficacy and safety.

Trial registration: Clinicaltrials.org Identifier: NCT02954172. Registered on 3 November 2016. Https:// clinicaltrials.gov/.

Keywords: Bevacizumab; IBI305; non-small cell lung cancer (NSCLC); biosimilar; vascular endothelial growth factor (VEGF)

Submitted Sep 30, 2019. Accepted for publication Nov 15, 2019.

doi: $10.21037 /$ tlcr.2019.12.23

View this article at: http://dx.doi.org/10.21037/tlcr.2019.12.23

\section{Introduction}

Bevacizumab is a monoclonal antibody (mAb) that targets vascular endothelial growth factor (VEGF) and suppresses tumor growth (1). The antitumor efficacy of bevacizumab combined with various chemotherapy regimens has been proven and supported by numerous large-scale phase 3 randomized controlled clinical trials (2). Subsequently, bevacizumab was approved with combination therapies to treat different cancers including metastatic colorectal, metastatic or recurrent non-squamous non-small-cell lung cancer (NSCLC), metastatic renal cell cancer, recurrent glioblastoma (US only), metastatic breast cancer (Europe only), persistent, recurrent, or metastatic cervical cancer, and recurrent epithelial ovarian, fallopian tube and primary peritoneal cancers $(3,4)$. It was approved in China as firstline treatment in combination with platinum-doublet chemotherapy for advanced or recurrent non-squamous NSCLC in 2015.

In recent years, value-based cancer care has become a hot topic in oncology, which focuses on how to assess effectively the value of various cancer treatments (5-7). The American Society of Clinical Oncology (ASCO) emphasizes not only the efficacy of drugs and regimens but also their price, taken cost-effectiveness into account. The National Comprehensive Cancer Network (NCCN) Guidelines also launched the "Evidence Blocks" version to provide more 
comprehensive guidance to evaluate the affordability of various drugs and treatment regimens $(8,9)$.

Research and development into biosimilar agents is an important means to ensure the efficacy as the reference drug while providing affordable price. A biosimilar is a drug that has comparable activity to a reference biological product that has been licensed for marketing. No clinical differences in purity, efficacy or safety between the reference drug and the biosimilar are permitted; however, small changes to inactive components of the product are allowed $(10,11)$. Biosimilar products provide an affordable alternative therapy for patients.

IBI305 is a bevacizumab biosimilar candidate and a previous clinical study in healthy males has shown that IBI305 was similar to the reference bevacizumab in terms of its pharmacokinetic profile, immunogenicity and safety profile (12). The aims of the present study were to confirm the therapeutic similarity of IBI305 to the reference bevacizumab with regard to its safety and efficacy as firstline therapy in patients with advanced or metastatic nonsquamous NSCLC.

\section{Methods}

\section{Study design and patients}

The study was a randomized, double-blind, multicenter, phase 3 clinical trial of NSCLC patients recruited from 42 centers in China (Clinicaltrials.org Identifier: NCT02954172). The study design followed the technical guidance issued by the regulatory authority in China [National Medical Products Administration (NMPA)] responsible for the development and evaluation of biosimilars, of which the requirement for the choice of study participants, study design and endpoints are similar to the European Commission, the European Medicines Agency (EMA) and the United States (US) Food and Drug Administration (FDA) requirements. The study followed the Declaration of Helsinki and Good Clinical Practice. The ethics committees of each study center approved the protocol and amendments; signed consent forms were provided by every patient that took part in the clinical trial.

Patients aged 18 to 75 years with unresectable locally advanced (stage IIIB), metastatic (stage IV) or recurrent non-squamous NSCLC, as confirmed by histological and/or cytological analysis, were enrolled. Major criteria for inclusion were: at least one measurable lesion as defined by the Response Evaluation Criteria in Solid
Tumor (RECIST version 1.1) criteria; Eastern Tumor Collaborative Group Performance Status score (ECOGPS) 0 or 1 ; satisfactory hepatic, renal, and bone marrow functions; and life expectancy $\geq 6$ months. Major exclusion criteria were: combined non-small cell or small cell cancer or adeno-squamous cancer with squamous cells as the main component; epidermal growth factor receptor (EGFR) sensitive mutations; previously treated with systemic anticancer therapy; and relapse within 6 months after adjuvant therapy. The comprehensive details about the exclusion and inclusion criteria are described in the protocol in http:// fp.amegroups.cn/cms/1c6c7f497a01b395836c8860c6fd8d 5f/tlcr.2019.12.23-1.pdf.

\section{Methodology}

\section{Randomization and masking}

Eligible patients were randomly assigned (1:1) to the IBI305 or bevacizumab treatment groups. A centralized randomization procedure with pre-allocated blocks was used. Patients were stratified based on age ( $<60 v s$. $\geq 60$ years) and EGFR status (wild-type $v$ s. unknown type). Treatment allocation was blinded using an interactive web response system. Investigators, patients and individuals who performed the analyses and assessments were blinded until database lock. In each center, the study medication was prepared by a dedicated, independent, unblinded study nurse.

\section{Procedures}

Patients received a maximum of 6 cycles of intravenously (IV)-administered IBI305 or reference bevacizumab $(15 \mathrm{mg} / \mathrm{kg})$, combined with IV-administered carboplatin (the area under the curve was 6) and paclitaxel $\left(175 \mathrm{mg} / \mathrm{m}^{2}\right)$. Patients then received IV-administered IBI305 or bevacizumab $(7.5 \mathrm{mg} / \mathrm{kg})$ according to their original treatment assignment as maintenance therapy. Therapy was administered at 3 -week intervals until one or more of the following occurred: intolerable toxicity; consent withdrawal; disease progression; loss of follow-up; or death.

Objective response rate (ORR) was evaluated by an independent radiological review committee (IRRC) and an investigator based on expert computed tomography or magnetic resonance imaging assessments. Imaging examinations were conducted at baseline and then at 6-week intervals ( \pm 7 days) during the course of therapy. Overall responses were confirmed by two continuous complete responses (CR) or partial responses (PR) at intervals of at least 4 weeks. 


\section{Outcomes}

Confirmed ORR, based on the RECIST version 1.1 criteria by the IRRC was the primary efficacy endpoint. The secondary efficacy endpoints were duration of response (DOR), disease control rate (DCR), progression-free survival (PFS) and overall survival (OS).

The safety profiles were compared by adverse events (AEs) and immunogenicity. Pharmacokinetic and pharmacodynamic endpoints were the drug steady-state concentrations after multiple administrations, as well as concentrations of VEGF.

\section{Statistical analysis}

Statistical analyses were performed using the SAS Enterprise Guide (version 7.11).

Based on the assumption that $50 \%$ of patients would achieve objective response in both the IBI305 and bevacizumab groups, a cohort of 218 patients per group (436 in total) would provide approximate $80 \%$ power to confirm the clinical equivalence in ORR between IBI305 and bevacizumab groups, at a predefined equivalence margin $(0.75,1.33)$ for the $90 \%$ CI of the ORR ratio (IBI305/ bevacizumab).

Clinical equivalence was confirmed if $90 \%$ CI of the ORR ratio between 2 groups was within the predefined equivalence margin $(0.75,1.33)$. A generalized linear model including treatment groups and stratification factors was used to estimate the ORR ratio and its 90\% CI. The primary endpoint was analyzed in the full analysis set (FAS), including all randomized and evaluable patients who received at least one dose of IBI305 or bevacizumab. The intention-to-treat (ITT) and per-protocol (PP) sets were also used for the sensitivity analysis of the primary endpoint. All randomized patients were included in the ITT set, and patients in the FAS who were compliant with the protocol were included in the PP set.

Kaplan-Meier analysis was conducted to estimate survival curves and median PFS, DOR, OS and the 95\% CIs. A stratified Cox model was used to estimate the hazard ratios and the $90 \%$ CI between the 2 groups. The DCR was analyzed with the same method for ORR.

The AEs were coded following the Medical Dictionary for Regulatory Activities and graded according to the Common Terminology Criteria for Adverse Events (version 4.03).

\section{Results}

From November 28, 2016 to May 23, 2018, 450 patients were enrolled and randomly assigned to the IBI305 group $(\mathrm{N}=224)$ or the bevacizumab group $(\mathrm{N}=226)$. All 450 enrolled patients were included in the ITT set, 441 patients in the FAS (221 in the IBI305 group and 220 in the bevacizumab group), and 433 patients in the PP set (216 in the IBI305 group and 217 in the bevacizumab group). All 450 enrolled patients were treated hence all were included in the safety analysis. The primary analysis was conducted based on the data obtained by September 19, 2018. Patient distributions were similar in the 2 groups (Figure 1). The baseline characteristics and demographics of patients in the 2 groups were closely matched (Table 1).

\section{Efficacy}

The primary efficacy analysis was based on the FAS ( $\mathrm{N}=441)$. The confirmed ORRs in the IBI305 and bevacizumab groups reviewed by IRRC were $44.3 \%$ and $46.4 \%$, respectively. The ORR ratio was 0.95 (90\% CI: 0.803 to $1.135)$, within the predefined equivalence margin of 0.75 to 1.33 (Table 2). ORR ratios in the ITT and PP sets were 0.95 (90\% CI: 0.798 to 1.129 ) and 0.96 (90\% CI: 0.807 to 1.145 ), respectively, which also fell within the equivalence margin (Table S1). The ORR ratios were within the predefined equivalence margins across all subgroups, including age $(<60$ or $\geq 60$ years), EGFR status (wild-type or unknown), gender (male or female), ECOG scores (0 or 1), smoking status (current, former or never smoked), clinical stage (IIIB or IV) and recurrence status (yes or no) (Figure S1).

The DCRs of the IBI305 and bevacizumab groups determined by IRRC were $90.5 \%$ and $87.7 \%$, respectively. The DCR ratio was 1.03 (90\% CI: 0.975 to 1.090 ) (Table 2). The median DOR was 7.3 months for IBI305 and 6.9 months for bevacizumab $(\mathrm{P}=0.559)$.

A follow-up analysis was conducted based on the data obtained by May 22, 2019. With a median follow-up of 14.5 months, $78.3 \%$ of patients in the IBI305 group and $77.3 \%$ in the bevacizumab group reached progression or death events. The median PFS was 7.64 months for IBI305 and 7.77 months for bevacizumab $(\mathrm{P}=0.9987)$. There were $43.1 \%$ patients deceased, and there was no significant difference in 12 -month $(69.9 \%$ vs. $72.2 \%)$ and 18 -month (53.1\% vs. 53.9\%) OS rates between IBI305 and 




*Not all patients who completed 6 cycles of combination treatment entered into the maintenance phase and not all patients who received maintenance treatment completed 6 cycles of combination treatment.

Figure 1 Flowchart of patient enrollment, distribution, and discontinuation of treatment. 
Table 1 Baseline characteristics, tumor classification, and status between the treatment groups

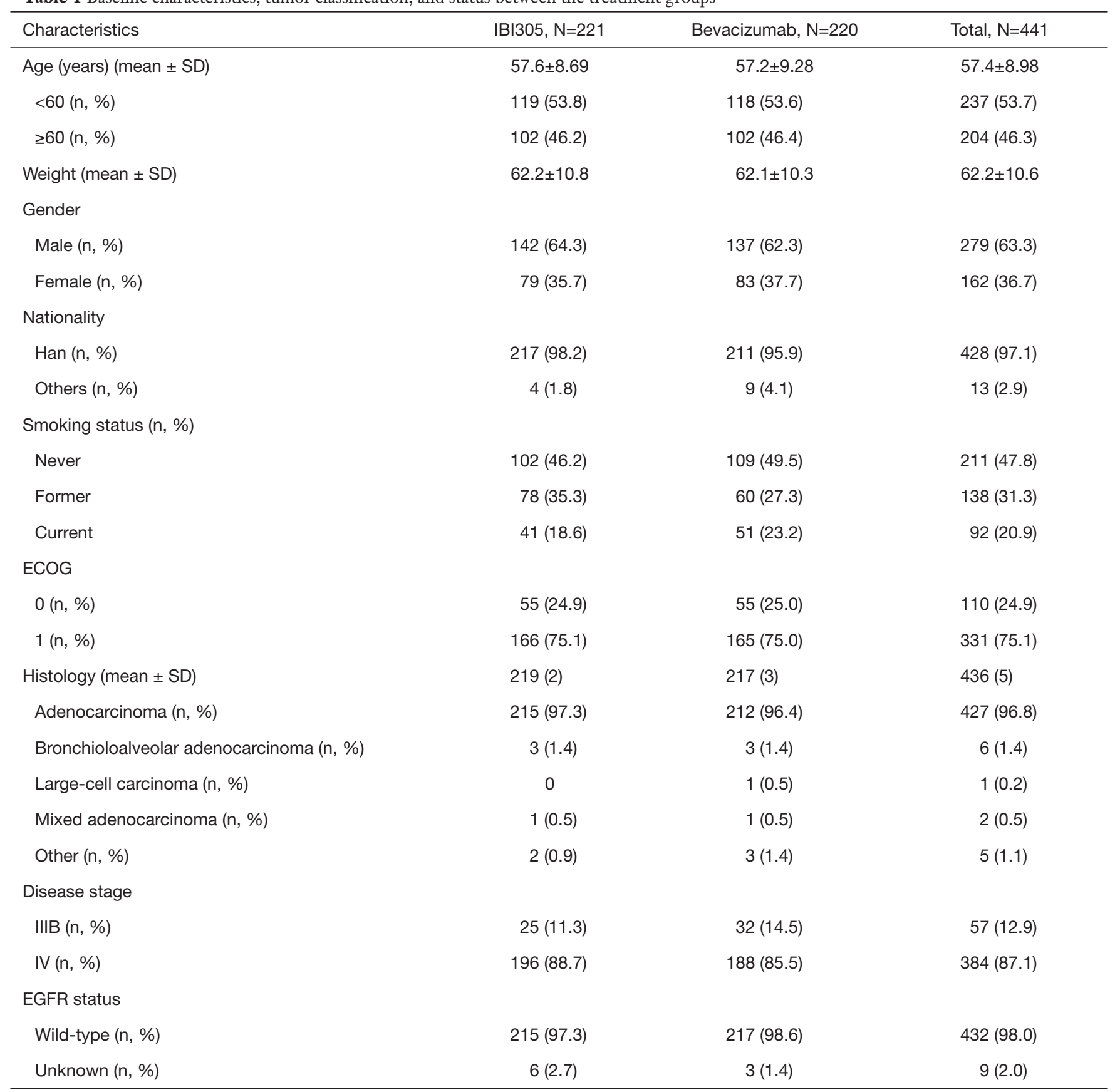

SD, standard deviation; ECOG, Eastern Cooperative Oncology Group; EGFR, epidermal growth factor receptor.

bevacizumab groups. By the cut-off date, fewer than $50 \%$ patients had deceased, therefore the OS may change in later analyses. There were no statistically significant differences in the median PFS and median OS between 2 treatment groups (Figure 2).

\section{Pharmacokinetic and pharmacodynamic analyses}

Pharmacokinetic data were analyzed in 76 patients in the IBI305 group and 75 in the bevacizumab group. The mean minimum concentrations in cycle 5 were $102.21 \mathrm{mg} / \mathrm{L}$ for IBI305 and $115.75 \mathrm{mg} / \mathrm{L}$ for bevacizumab. The trends of 
Table 2 Response evaluation (by the IRRC) in the FAS

\begin{tabular}{lccc}
\hline Treatment responses & IBI305 (N=221) & Bevacizumab (N=220) & Ratio (90\% Cl) \\
\hline Objective response rate (ORR) & $98(44.3 \%)$ & $102(46.4 \%)$ & $0.95(0.803,1.135)$ \\
Disease control rate (DCR) & $200(90.5 \%)$ & $193(87.7 \%)$ & $1.03(0.975,1.090)$ \\
Complete response (CR) & 0 & $102(46.4 \%)$ \\
Partial response (PR) & $98(44.3 \%)$ & $91(41.4 \%)$ \\
Stable disease (SD) & $102(46.2 \%)$ & $14(6.4 \%)$ \\
Disease progression (PD) & $11(5.0 \%)$ & $13(5.9 \%)$ \\
Unable to evaluate (NE) & $10(4.5 \%)$ & & \\
\hline
\end{tabular}

IRRC, independent radiological review committee; FAS, full analysis set; $\mathrm{Cl}$, confidential interval.

concentration changes were consistent between IBI305 and bevacizumab after multiple administrations (Figure $S 2 A$ ).

Pharmacodynamic data were analyzed in 76 patients in both the 2 groups. The changes of VEGF concentrations in plasma from baseline were similar between the 2 groups (Figure S2B), showing that the 2 groups exhibited consistent trends in pharmacodynamics due to drug-induced changes in plasma VEGF concentrations.

\section{Safety}

The incidence of AEs was 100\% in both treatment groups. There were no statistically significant differences between the IBI305 and bevacizumab groups in the incidence of AEs $\geq$ grade 3 (84.4\% vs. $89.8 \%$ ), serious AEs (33.5\% vs. $37.6 \%$ ), fatal AEs (2.7\% vs. 2.2\%), or AEs of special interest (AESIs) (53.6\% vs. 62.4\%) (all $\mathrm{P}>0.05$ ) (Table S2).

The most frequent mAb-related AEs in both groups were decreased white blood cell counts (24.1\% vs. $20.8 \%)$, decreased neutrophil counts $(23.7 \%$ vs. $20.8 \%)$, and proteinuria $(23.2 \%$ vs. $20.8 \%)$. The most frequent $\mathrm{mAb}-$ related AEs $\geq$ grade 3 in the IBI305 and bevacizumab groups were decreased neutrophil counts (16.1\% vs. 11.9\%) and decreased white blood cell counts $(9.8 \%$ vs. $6.6 \%)$ (Table 3). No significant differences were found between the 2 treatment groups in the incidences of AESIs regarding hemorrhage, proteinuria, hypertension, thromboembolic events, infusion reactions, congestive heart failure and fistulae (Figure S3).

In the IBI305 group, $2(0.9 \%)$ patients tested positive for anti-drug antibodies (ADA), 1 at baseline and the other at the end of treatment. In the bevacizumab group, $2(0.9 \%)$ patients tested positive for ADA at baseline. Neutralizing antibodies were not detected in patients in either treatment group.

\section{Discussion}

The 'Considerations for clinical research design of bevacizumab biosimilar' issued by NMPA recommends to conduct efficacy comparison in metastatic or recurrent non-squamous NSCLC with an ORR as primary endpoint and 0.75 to $1 / 0.75$ as equivalence margin. Therefore, the clinical equivalence between the IBI305 and bevacizumab was confirmed by an ORR ratio of 0.95 (90\% CI: 0.803 to $1.135)$, within the predefined equivalence margin of 0.75 to 1.33. Efficacy equivalence between IBI 305 and bevacizumab was also supported by subgroup and sensitivity analyses results in the ITT and PP sets.

Bevacizumab maintenance therapy is a worldwide standard after bevacizumab in combination with chemotherapy for non-squamous NSCLC patients with responsive or stable disease (13). Although ORR is recommended as a sensitive efficacy endpoint in biosimilar studies for anticancer products (11), it is indisputable that long-term observation could provide further confirmation of efficacy for biosimilars. Our study is the first to report a mature PFS result with a median follow-up of 14.5 months and $77.8 \%$ of patients achieved progression or death events by the study data cutoff. No statistical difference was observed between these 2 groups with a median PFS of 7.64 months for IBI305 and 7.77 months for bevacizumab $(\mathrm{P}=0.9987)$. The results observed in the IBI305 group were also comparable to previous studies of bevacizumab, including the BEYOND study (median PFS, 8.3 months for the EGFR wild-type subgroup) (14), AVAiL study Asian subgroup (median PFS, 8.2 months) $(15,16)$, and SAiL study Asian subgroup (median time to progression, 8.3 months) (17), 

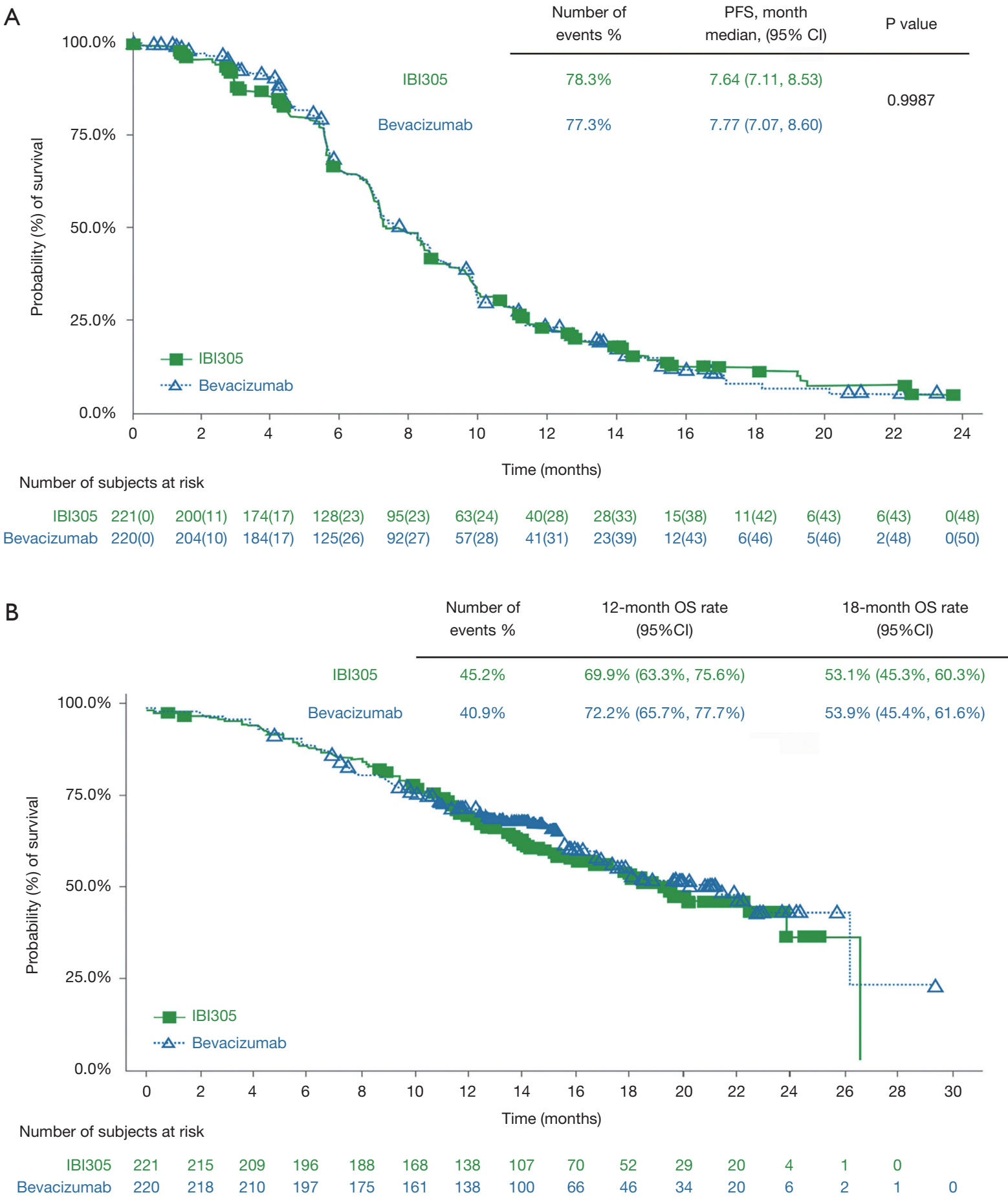

Figure 2 Long-term efficacy comparison between two groups. (A) Kaplan-Meier estimate of progression-free survival; (B) Kaplan-Meier estimate of overall survival. 
Table 3 Comparison of incidences $\geq 10 \%$ of $\mathrm{mAb}$-associated AEs between the treatment groups



$\mathrm{mAb}$, monoclonal antibody; AEs, adverse events.

which further confirmed the clinical equivalence of IBI305 with bevacizumab.

The incidence of EGFR mutations in patients with nonsquamous NSCLC is $40-50 \%$ in the Asian population (18). Several large-scale phase 3 clinical trials have confirmed that EGFR-tyrosine kinase inhibitors (TKIs) can significantly improve PFS and the quality of life in EGFR-mutant advanced NSCLC patients compared with conventional chemotherapy (19-24). Therefore, NCCN, ESMO and CSCO guidelines recommend EGFR-TKIs as the firstline treatment for these patients $(9,13,25)$. For the EGFR wild-type population, paclitaxel/carboplatin combined with bevacizumab is deemed as the standard first-line treatment. Additionally, it was found that PFS was longer in patients with EGFR mutations than in those with wild-type EGFR (12.4 vs. 8.3 months) when they received bevacizumab plus chemotherapy as fist-line treatment in the Beyond study (14). It is likely to overestimate the efficacy of bevacizumab by including patients with EGFR mutations. Notably, unlike previous studies, patients with EGFR activating mutations were excluded from the present study. The baseline characteristics showed that the vast majority of the patients (98\%) were wild-type in EGFR status. Thus the results of this study may better reflect the actual clinical efficacy of IBI305.

There were no significant differences between treatment groups in terms of the types, incidence and severity of AEs. The toxicity spectrum was consistent with the known safety profile of bevacizumab with no unexpected AEs were observed in the IBI305 group. No significant differences were reported between the 2 treatment groups in the incidence of AESIs including hemorrhage, proteinuria, hypertension, thromboembolic events, infusion reactions, congestive heart failure and fistulae. The pharmacokinetic and pharmacodynamic profiles between IBI305 and bevacizumab were similar. In addition, the immunogenicity profile was low $(<1 \%)$ and similar in the 2 groups.

Although the combination of bevacizumab and chemotherapy improves the therapeutic efficacy, the high price of bevacizumab is a factor that must be considered in clinical practice. According to the ASCO estimation, the addition of bevacizumab to the paclitaxel/carboplatin for NSCLC results in an incremental cost of $\$ 11,726$ per month (26). In China, the addition of bevacizumab to the treatment regimen for NSCLC results in an incremental cost-effectiveness ratio of $\$ 299,155$ per quality-adjusted life year. This cost greatly exceeds the accepted Chinese society willingness-to-pay level of $\$ 23,970$ (27). Therefore, the NCCN Guidelines (2019 version) ranked the combination of bevacizumab and chemotherapy as expensive to very expensive regimens, the same as the ranking of pembrolizumab combined with chemotherapy (9). Studies have shown that the high cost and insufficient medical insurance support are known barriers to comprehensive adoption of bevacizumab in clinical practice (28). EU biosimilar monitoring broadly concerned the safety and 
efficacy of their generic drugs using in individual countries. General, the discounts of biosimilar drugs range from $20 \%$ to $35 \%$ of their reference products (29). In China, the price of biosimilars were reported as $60 \%$ lower than reference products (30). Hence the development of effective and safe biosimilars will provide greater access for patients and will lower costs for these life-saving treatments. One bevacizumab biosimilar (ABP 215) has been approved by FDA and EMA. However, currently no bevacizumab biosimilar has been approved by NMPA. Access to IBI305 will provide a cost-effective alternative treatment for patients and more feasibility for the exploration of novel treatment regimen.

\section{Conclusions}

In conclusion, the present multicenter, randomized, phase 3 study provides strong evidence of the clinical similarity of IBI305 to bevacizumab in terms of efficacy, safety and immunogenicity. This biosimilar drug provides a costeffective alternative treatment for patients with nonsquamous NSCLC.

\section{Acknowledgments}

We thank the patients and their families and the participating study teams for making this study possible and Yuan Fang (Innovent Biologics, Inc., China) for editorial assistance. The study was sponsored by Innovent Biologics, Inc.

\section{Footnote}

Conflicts of Interest: L Zhang received research grants from Eli Lily and Pfizer. H Zhou, J Yu and X Sun are staff of Innovent Biologics, Inc. The other authors have no conflicts of interest to declare.

Ethical Statement: The authors are accountable for all aspects of the work in ensuring that questions related to the accuracy or integrity of any part of the work are appropriately investigated and resolved. The ethics committees of each study center approved the protocol and amendments; signed consent forms were provided by every patient that took part in the clinical trial.

\section{References}

1. Kim KJ, Li B, Winer J, et al. Inhibition of vascular endothelial growth factor-induced angiogenesis suppresses tumour growth in vivo. Nature 1993;362:841-4.

2. Ahmadizar F, Onland-Moret NC, de Boer A, et al. Efficacy and Safety Assessment of the Addition of Bevacizumab to Adjuvant Therapy Agents in Cancer Patients: A Systematic Review and Meta-Analysis of Randomized Controlled Trials. PLoS One 2015;10:e0136324.

3. Avastin (bevacizumab) Prescribing Information. Available online: https://www.accessdata.fda.gov/drugsatfda_docs/ label/2016/125085s317lbl.pdf

4. Agency EM. Avastin (Summary of Product Characteristics). 2018.

5. Young RC. Value-Based Cancer Care. N Engl J Med 2015;373:2593-5.

6. Alvarnas J, Majkowski GR, Levine AM. Moving Toward Economically Sustainable Value-Based Cancer Care in the Academic Setting. JAMA Oncol 2015;1:1221-2.

7. Djulbegovic B. Value-Based Cancer Care and the Excessive Cost of Drugs. JAMA Oncol 2015;1:1301-2.

8. Carlson RW, Jonasch E. NCCN Evidence Blocks. J Natl Compr Canc Netw 2016;14:616-9.

9. (NCCN) NCCN. Clinical Practice Guidelines in Oncology. Non-Small Cell Lung Cancer Evidence Blocks ${ }^{\mathrm{TM}}$, Version 3. Available online: https://www.ncen. org/professionals/physician_gls/pdf/nscl_blocks.pdf. 2019.

10. (FDA) UFaDA. CfDEaR. Scientific considerations in demonstrating biosimilarity to a reference product. Guidance for Industry 2015, Available online: https://www. federalregister.gov/documents/2015/04/30/2015-10062/ scientific-considerations-in-demonstrating-biosimilarityto-a-reference-product-guidance-for

11. European Medicines Agency. Guideline on similar biological medicinal products containing biotechnologyderived proteins as active substance: nonclinical and clinical issues 2015. Available online: https:// www.ema.europa.eu/documents/scientific-guideline/ guideline-similar-biological-medicinal-productscontaining-biotechnology-derived-proteins-active_en-2. pdf. 2015.

12. Zhang $\mathrm{H}$, Zhu X, Wei H, et al. A phase I, randomized, double-blinded, single-dose study evaluating the pharmacokinetic equivalence of the biosimilar IBI305 and bevacizumab in healthy male subjects. Int J Clin 
Pharmacol Ther 2019;57:167-74.

13. Planchard D, Popat S, Kerr K, et al. Metastatic non-small cell lung cancer: ESMO Clinical Practice Guidelines for diagnosis, treatment and follow-up. Ann Oncol 2018;29:iv192-237.

14. Zhou C, Wu YL, Chen G, et al. BEYOND: A Randomized, Double-Blind, Placebo-Controlled, Multicenter, Phase III Study of First-Line Carboplatin/ Paclitaxel Plus Bevacizumab or Placebo in Chinese Patients With Advanced or Recurrent Nonsquamous NonSmall-Cell Lung Cancer. J Clin Oncol 2015;33:2197-204.

15. Reck M, von Pawel J, Zatloukal P, et al. Overall survival with cisplatin-gemcitabine and bevacizumab or placebo as first-line therapy for nonsquamous non-small-cell lung cancer: results from a randomised phase III trial (AVAiL). Ann Oncol 2010;21:1804-9.

16. Reck M, von Pawel J, Zatloukal P, et al. Phase III trial of cisplatin plus gemcitabine with either placebo or bevacizumab as first-line therapy for nonsquamous nonsmall-cell lung cancer: AVAil. J Clin Oncol 2009;27:1227-34.

17. Mok TS, Hsia TC, Tsai CM, et al. Efficacy of bevacizumab with cisplatin and gemcitabine in Asian patients with advanced or recurrent non-squamous non-small cell lung cancer who have not received prior chemotherapy: a substudy of the Avastin in Lung trial. Asia Pac J Clin Oncol 2011;7 Suppl 2:4-12.

18. Gou LY, Wu YL. Prevalence of driver mutations in nonsmall-cell lung cancers in the People's Republic of China. Lung Cancer (Auckl) 2014;5:1-9.

19. Mok TS, Wu YL, Thongprasert S, et al. Gefitinib or carboplatin-paclitaxel in pulmonary adenocarcinoma. $\mathrm{N}$ Engl J Med 2009;361:947-57.

20. Fukuoka M, Wu YL, Thongprasert S, et al. Biomarker analyses and final overall survival results from a phase III, randomized, open-label, first-line study of gefitinib versus carboplatin/paclitaxel in clinically selected patients with advanced non-small-cell lung cancer in Asia (IPASS). J Clin Oncol 2011;29:2866-74.

21. Zhou C, Wu YL, Chen G, et al. Erlotinib versus chemotherapy as first-line treatment for patients with advanced EGFR mutation-positive non-small-cell lung cancer (OPTIMAL, CTONG-0802): a multicentre, open-label, randomised, phase 3 study. Lancet Oncol 2011;12:735-42.

22. Sequist LV, Yang JC, Yamamoto N, et al. Phase III study of afatinib or cisplatin plus pemetrexed in patients with metastatic lung adenocarcinoma with EGFR mutations. J Clin Oncol 2013;31:3327-34.

23. Wu YL, Zhou C, Hu CP, et al. Afatinib versus cisplatin plus gemcitabine for first-line treatment of Asian patients with advanced non-small-cell lung cancer harbouring EGFR mutations (LUX-Lung 6): an open-label, randomised phase 3 trial. Lancet Oncol 2014;15:213-22.

24. Yang JC, Wu YL, Schuler $M$, et al. Afatinib versus cisplatin-based chemotherapy for EGFR mutation-positive lung adenocarcinoma (LUX-Lung 3 and LUX-Lung 6): analysis of overall survival data from two randomised, phase 3 trials. Lancet Oncol 2015;16:141-51.

25. Oncology CSoC. CSCO Primary Diagnosis and Treatment Guidelines for Lung Cancer. Beijing 2017.

26. Schnipper LE, Davidson NE, Wollins DS, et al. American Society of Clinical Oncology Statement: A Conceptual Framework to Assess the Value of Cancer Treatment Options. J Clin Oncol 2015;33:2563-77.

27. Zheng H, Xie L, Zhan M, et al. Cost-effectiveness analysis of the addition of bevacizumab to chemotherapy as induction and maintenance therapy for metastatic nonsquamous non-small-cell lung cancer. Clin Transl Oncol 2018;20:286-93.

28. Monk BJ, Lammers PE, Cartwright T, et al. Barriers to the Access of Bevacizumab in Patients with Solid Tumors and the Potential Impact of Biosimilars: A Physician Survey. Pharmaceuticals (Basel) 2017. doi: 10.3390/ ph10010019.

29. Bennett CL, Chen B, Hermanson T, et al. Regulatory and clinical considerations for biosimilar oncology drugs. Lancet Oncol 2014;15:e594-605.

30. Miller P, Wang F, Kubavat H. Biosimilars in China. Available online: http:// www.pmline.com/pharma_ intelligence/biosimilars_in_china460090. PMLiVE. 2013.

Cite this article as: Yang Y, Wu B, Huang L, Shi M, Liu Y, Zhao Y, Wang L, Lu S, Chen G, Li B, Xie C, Fang J, Yang N, Zhang Y, Cui J, Song Y, Zhang C, Mei X, Cao B, Yang L, Cheng Y, Ying K, Sun T, Ren B, Yu Q, Liao Z, Pei Z, Wang M, Zhou J, Yu S, Feng G, Wan H, Wang H, Gao S, Wang J, An G, Geng Y, Ji Y, Yuan Y, Ma S, Jia Z, Hu M, Zhou H, Yu J, Sun X, Zhang L. Biosimilar candidate IBI305 plus paclitaxel/ carboplatin for the treatment of non-squamous non-small cell lung cancer. Transl Lung Cancer Res 2019;8(6):989-999. doi: 10.21037/tlcr.2019.12.23 


\section{Supplementary}

Table S1 Response evaluation (by the IRRC) in the ITT and PP sets

\begin{tabular}{|c|c|c|c|}
\hline Treatment responses & IBI305 (n, \%) & Bevacizumab (n, \%) & ORR ratio $(90 \% \mathrm{Cl})$ \\
\hline Objective response rate (ORR) & $98(43.8)$ & $104(46.0)$ & $0.95(0.798,1.129)$ \\
\hline Complete response (CR) & 0 & 0 & \\
\hline Partial response (PR) & $98(43.8)$ & $104(46.0)$ & \\
\hline Disease progression (PD) & $11(4.9)$ & $15(6.6)$ & \\
\hline Unable to evaluation (NE) & $13(5.8)$ & $16(7.1)$ & \\
\hline Number of patients in PP set & 216 & 217 & \\
\hline Objective response rate (ORR) & $96(44.4)$ & $100(46.1)$ & $0.96(0.807,1.145)$ \\
\hline Stable disease (SD) & $101(46.8)$ & $90(41.5)$ & \\
\hline Disease progression (PD) & $11(5.1)$ & $14(6.5)$ & \\
\hline Unable to evaluate (NE) & $8(3.7)$ & $13(6.0)$ & \\
\hline
\end{tabular}

IRRC, independent radiological review committee; ITT, intention to treat; PP, per protocol; Cl, confidential interval; ORR, objective response rate.

Subgroup (number of participants)
Total $(n=441)$
Age $(y r)$
$<60(n=237)$
$\geq 60(n=204)$
EGFR performance status
Wild $(n=432)$
Unknown $(n=9)$
Gender
Male $(n=279)$
Female $(n=162)$
ECOG score
O $(n=110)$
1 ( $n=331)$
Smoke
Yes $(n=92)$
No $(n=211)$
Quitted $(n=138)$
Tumor clinical stages
IIIB $(n=57)$
IV $(n=384)$
Recurrence
Yes $(n=36)$
No $(n=405)$

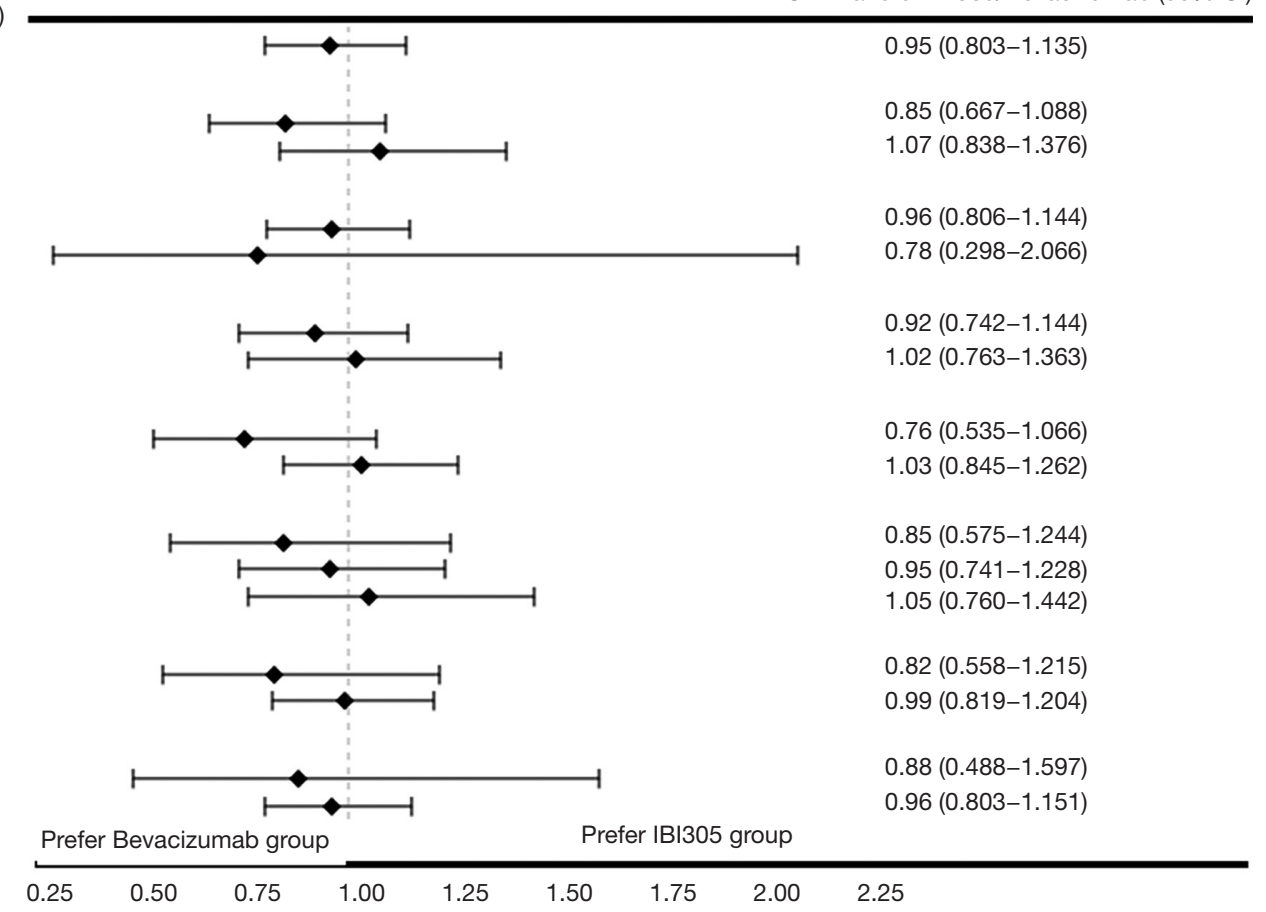

Figure S1 ORR subgroup analyses. ORR, objective response rate. 
A

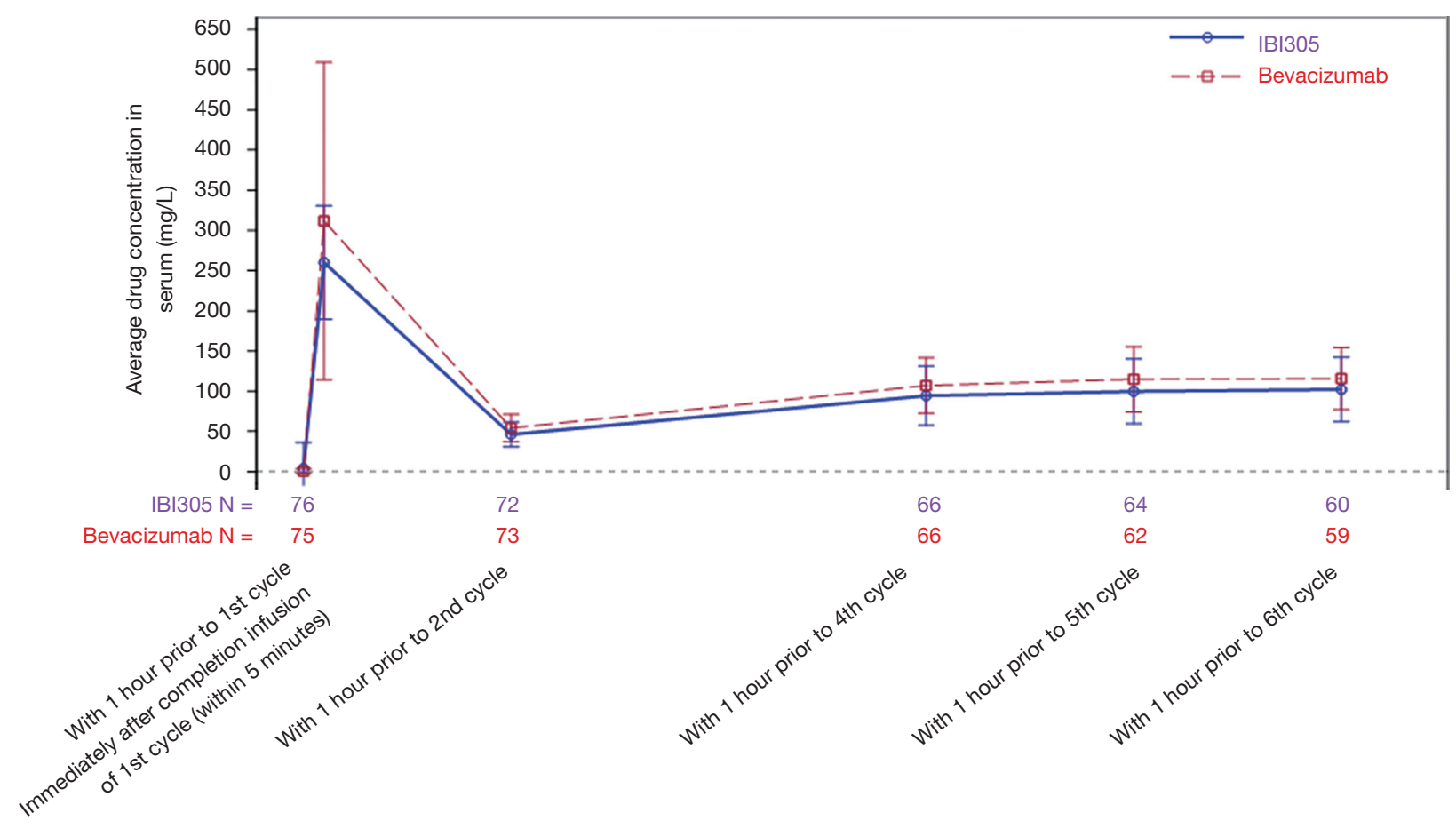

B

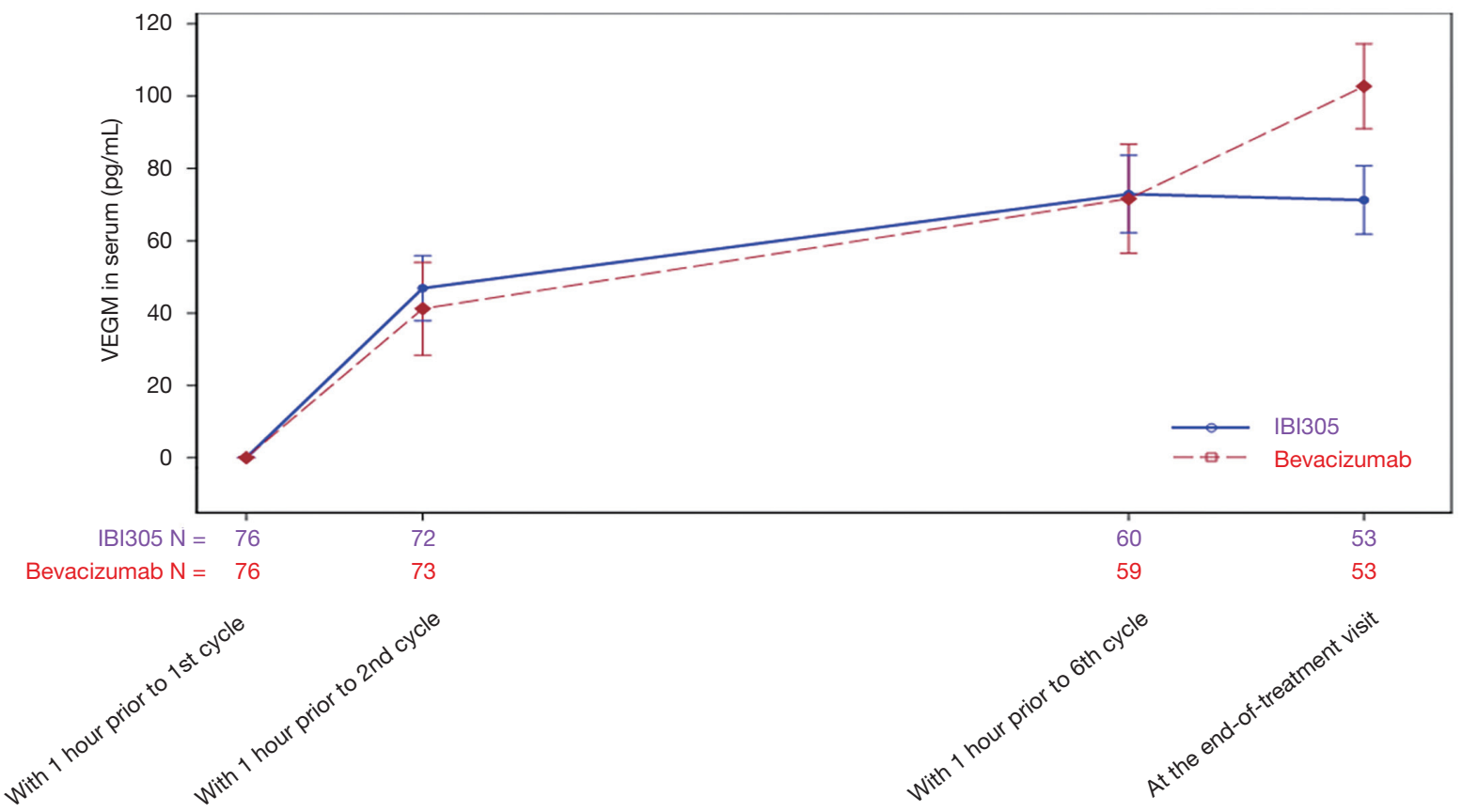

Figure S2 (A) Blood concentrations of IBI305 and bevacizumab over time, and (B) changes in plasma VEGF concentrations compared to baseline at different time points. Bars are SDs. VEGF, vascular endothelial growth factor; SD, standard deviation. 
Table S2 Overall summary of adverse events (AE)

\begin{tabular}{|c|c|c|c|}
\hline AE type & IBI305 (n, \%) & Bevacizumab (n, \%) & $P$ value \\
\hline Any $A E$ related with $m A b$ & $173(77.2)$ & $181(80.1)$ & 0.460 \\
\hline Any $\mathrm{AE}$ related with chemotherapy & $220(98.2)$ & $225(99.6)$ & 0.363 \\
\hline $\mathrm{AE}$ grade $\geq 3^{\#}$ & $189(84.4)$ & $203(89.8)$ & 0.085 \\
\hline Fatal AEs & $6(2.7)$ & $5(2.2)$ & 0.749 \\
\hline AEs leading to termination of treatments & $13(5.8)$ & $8(3.5)$ & 0.255 \\
\hline AEs leading to transient $m A b$ discontinuation & $99(44.2)$ & $102(45.1)$ & 0.842 \\
\hline AEs leading to transient discontinuation of chemotherapeutic drug & $99(44.2)$ & $97(42.9)$ & 0.785 \\
\hline AESIs & $120(53.6)$ & $141(62.4)$ & 0.0581 \\
\hline
\end{tabular}

\#, according to the Common Terminology Criteria for Adverse Events (CTCAE), version 4.03. mAb, monoclonal antibody; SAE, serious adverse event; AESIs, adverse events of special interest.

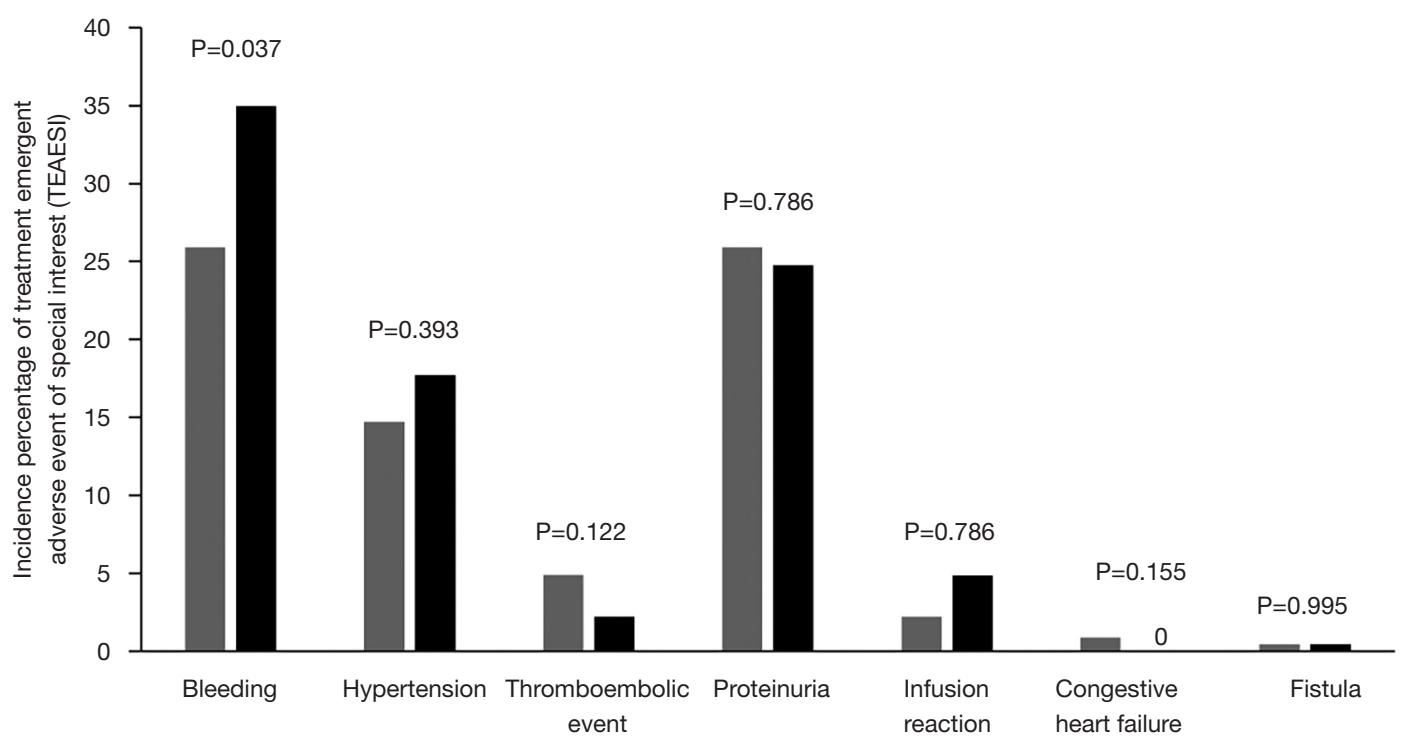

Figure S3 Comparison of the incidence of AESIs in the IBI305 and bevacizumab groups. AESI, adverse events of special interest. 